\title{
DESENVOLVIMENTO DE FERRAMENTA COMPUTACIONAL UTILIZANDO MICROTOMOGRAFIA COMPUTADORIZADA PARA APLICAÇÕES EM METROLOGIA
}

\author{
Julio Cesar Corrêa de OLIVEIRA ${ }^{1}$ \\ João Flávio Vieira de VASCONCELLOS ${ }^{2}$ \\ Joaquim Teixeira de ASSIS $^{3}$
}

\begin{abstract}
${ }^{1}$ Universidade do Estado do Rio de Janeiro, Instituto Politécnico, 28630-050 - Nova Friburgo, RJ, Brasil. jcoliveira.prof@yahoo.com.br

${ }^{2}$ Universidade do Estado do Rio de Janeiro, Instituto Politécnico, 28630-050 - Nova Friburgo, RJ, Brasil. jflavio@iprj.uerj.br

${ }^{3}$ Universidade do Estado do Rio de Janeiro, Instituto Politécnico, 28630-050 - Nova Friburgo, RJ, Brasil. joaquim@iprj.uerj.br
\end{abstract}

Recebido em: 30/05/2014 - Aprovado em: 20/09/2014 - Disponibilizado em: 15/12/2014

\begin{abstract}
Resumo. Este artigo apresenta um estudo sobre a utilização da Microtomografia Computadorizada ( $\mu$ CT) aplicada à metrologia focando no desenvolvimento de uma ferramenta computacional. Para isso analisa as principais técnicas de processamento de imagens digitais para a detecção de bordas. É feita uma comparação dos resultados obtidos a partir de diferentes técnicas de detecção de borda, principalmente da primeira e da segunda derivadas, aplicadas a imagens tomográficas de elementos de formas geométricas simples e as medidas obtidas diretamente através da utilização de um paquímetro digital. Os resultados obtidos com a ferramenta computacional foram equivalentes ao obtidos com o paquímetro digital, demonstrando que as técnicas utilizadas podem contribuir para o desenvolvimento de uma ferramenta computacional aplicada a metrologia, principalmente, para o caso de objetos de dimensão reduzida, deformáveis ou mesmo para medição interna de forma não destrutiva.
\end{abstract}

Palavras-chave: Microtomografia; Metrologia; Detecção de Bordas; Matlab; Processamento de Imagens

\section{CONSIDERAÇÕES INICIAIS}

A tomografia computadorizada (CT) é uma técnica muito utilizada para diagnósticos por imagem na área médica. Consiste em produzir uma imagem, de uma fatia, do interior de um corpo ou objeto através da utilização de programas de computadores capazes de processar os dados coletados de atenuação de Raios-X que atravessam o corpo ou objeto examinado. A partir da obtenção de diversas fatias é possível também a reconstrução tridimensional desse corpo ou objeto.
Por se tratar de uma técnica que permite a visualização de interiores sem necessidade de invasão ou destruição a CT despertou interesse de muitas áreas além da médica, incluindo a indústria, que já a utiliza há mais de 30 anos para inspeção interna de materiais ou para ensaios não destrutivos.

Porém, as aplicações da CT vão além de simplesmente visualizar o interior do objeto, podendo ser utilizada, por exemplo, para realização de medidas. Tomógrafos médicos convencionais realizam medidas de partes internas de forma corriqueira. Entretanto, para a alguns setores da indústria 
a exatidão da medida é fator fundamental. $\mathrm{Na}$ área médica se um tumor de câncer for diagnosticado como tendo $2 \mathrm{~cm}$ ao invés de $2.001 \mathrm{~cm}$, de maneira geral, isto será irrelevante, mas para alguns setores como o eletrônico, de plásticos ou o automobilístico, por exemplo, pode ser crucial. A área, no ramo da indústria, que se preocupa com as mediadas é a Metrologia Industrial. Os sistemas de medição mais comuns utilizados são sistemas ópticos ou apalpadores após a destruição (corte em seção transversal) da peça. Os avanços da informática a partir do final do século XX início do XXI tem criado condições para o surgimento de soluções utilizando a CT em metrologia industrial. [2]

\section{TOMOGRAFIA COMPUTADORIZADA APLICADA A METROLOGIA}

Para metrologia, em geral, é utilizada a Microtomografia Computadorizada $(\mu \mathrm{CT})$ que é uma variante da tomografia convencional e surgiu com a necessidade de se inspecionar amostras com estruturas muito pequenas da ordem de micrômetros.

Estudos como [1] apontam que a $\mu \mathrm{CT}$, apesar de necessitar melhorias em relação à rastreabilidade das medidas, é o único sistema de medição que atende a todos os requisitos para peças de dimensões menores que $0,1 \mathrm{~m}$. Por isso, diversos estudos vêm sendo desenvolvidos para aperfeiçoar as técnicas de $\mu \mathrm{CT}$ para aplicações metrológicas.

Inúmeros fatores podem influenciar o resultado de um processo de medição através uCT, estes fatores envolvem o humano, as características construtivas do tomógrafo, ajustes do tomógrafo, as técnicas de reconstrução da imagem e a própria técnica de medição.

Existe no mercado tanto tomógrafos especificamente projetados para metrologia industrial quanto máquinas de medir por coordenadas que utilizam a CT para a melhoria de suas medidas, entretanto, estes são sistemas de custo elevado e tecnologia proprietária.

Portanto, este estudo se propõe, dentro do conjunto de fatores que podem influenciar no resultado das medidas, analisar os aspectos relativos ao processamento de imagens com o propósito de verificar a viabilidade do desenvolvimento de uma ferramenta computacional destinada a metrologia que possa ser utilizada em tomógrafos convencionais.

Para se realizar uma medida, o primeiro passo, óbvio, é saber onde realizar a medida. Contudo, este pode não ser tão simples assim. Para se realizar uma medida através de imagens digitais é preciso definir as bordas do objeto a ser medido. De acordo com [3] bordas são definidas "como a fronteira entre duas regiões cujos níveis de 
cinza predominantes são razoavelmente diferentes." Bordas são regiões de transição. O termo borda aqui se refere às bordas de luminosidade, entretanto, ressalta-se que de acordo com a necessidade podem ser definidos outros tipos de bordas como, por exemplo, textura ou cor.

As figuras (1), (2) e (3) correspondem, respectivamente, a imagem tomográfica de uma determinada peça e a linha central desta imagem e aos tons de cinza desta linha.

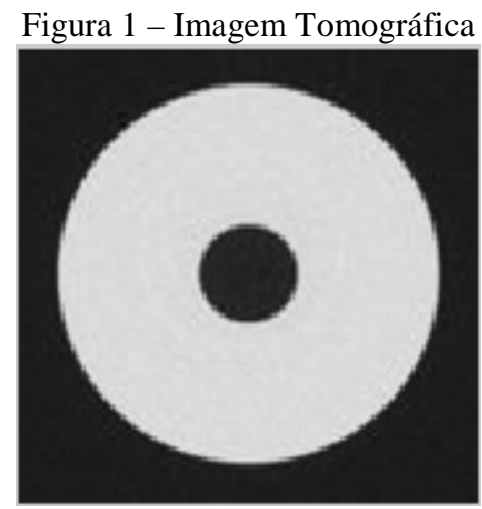

Figura 2 - Linha central (figura 1)

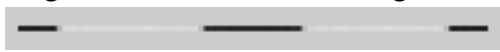

Figura 3 - Tons de Cinza - Linha central (figura 2)

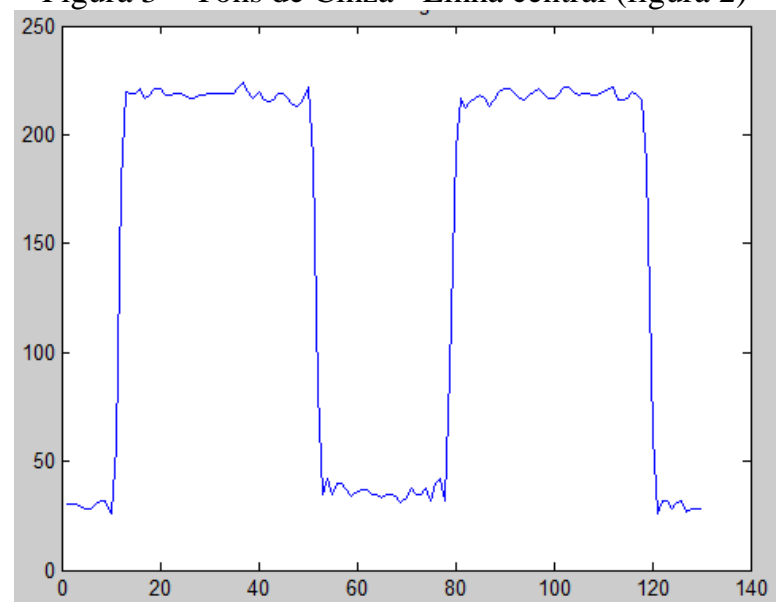

Observa-se na figura (3), como dito anteriormente, que a borda é uma região de transição, podendo ser observada a dificuldade de definir onde deve ser realizada a medição a fim de se obter a exatidão necessária a aplicações metrológicas.

\section{A FERRAMENTA COMPUTACIONAL}

Para o desenvolvimento da ferramenta computacional a fim de avaliar técnicas programação foi adotado o software Matlab ${ }^{\circledR}$ R2010a e utilizado objetos de geometria simples, como círculos ou retângulos.

Primeiramente, foi analisada a utilização a técnica de limiarização. A limiarização ou binarização é uma técnica de segmentação de imagens que utiliza um ponto de corte chamado de threshold. Valores menores a este ponto são representados por pixels pretos e valores maiores ou iguais por pixels brancos, por exemplo. Assim, a região de interesse pode ser a branca e a de não interesse a preta, figuras (6) e (7). A técnica mais simples consiste em determinar apenas um ponto de corte chamado de threshold global, porém é possível utilizar mais de um ponto de corte chamados de thresholds locais.

[5] destaca que apesar do threshold local ser o preferido para aplicações metrológicas não há uma unanimidade sobre o tema. Como se trata de objetos homogêneos, isto é, feitos de apenas um material, optou-se pelo threshold global. 
As figuras (6) e (7) exemplificam como a definição do threshold pode influenciar na medida. A definição do threshold é feita com base no histograma da imagem, figura (5). Para a escolha threshold foi adotado o algoritmo de Otsu [6] devido a sua reconhecida eficiência e facilidade de implementação.

Figura 4 - Imagem Tomográfica

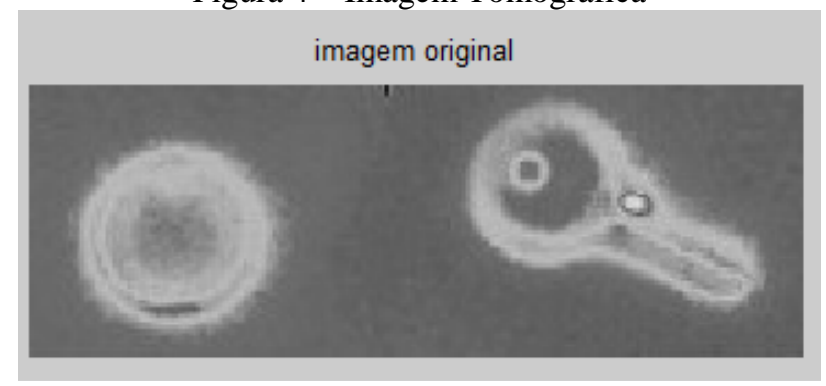

Figura 5 - Histograma (Imagem - Figura 4)

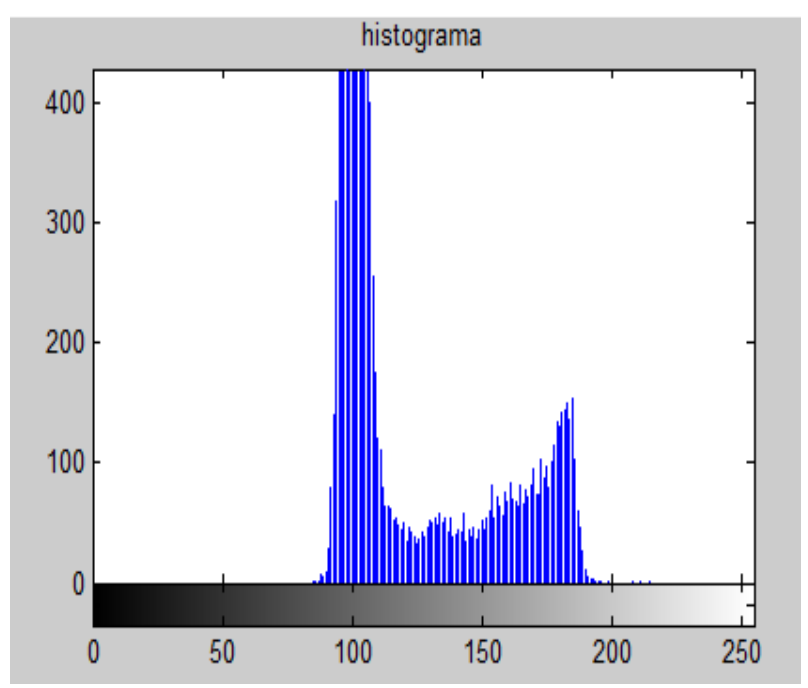

Figura 6 - Imagem binarizada

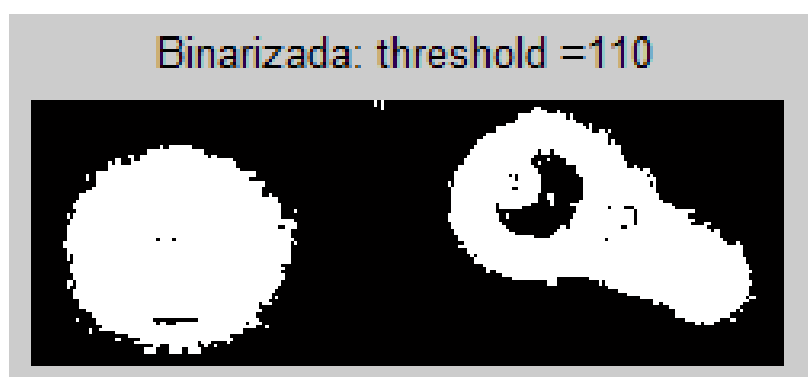

Outra técnica analisada foi à medição entre picos da $1^{\mathrm{a}}$ e $2^{\mathrm{a}}$ derivadas. As figuras (8) e (9) apresentam o respectivos resultados da $1^{\mathrm{a}}$ e $2^{\mathrm{a}}$ derivadas aplicadas a imagem da figura (2).

Figura $8-1^{\mathrm{a}}$ Derivada (Figura 2)

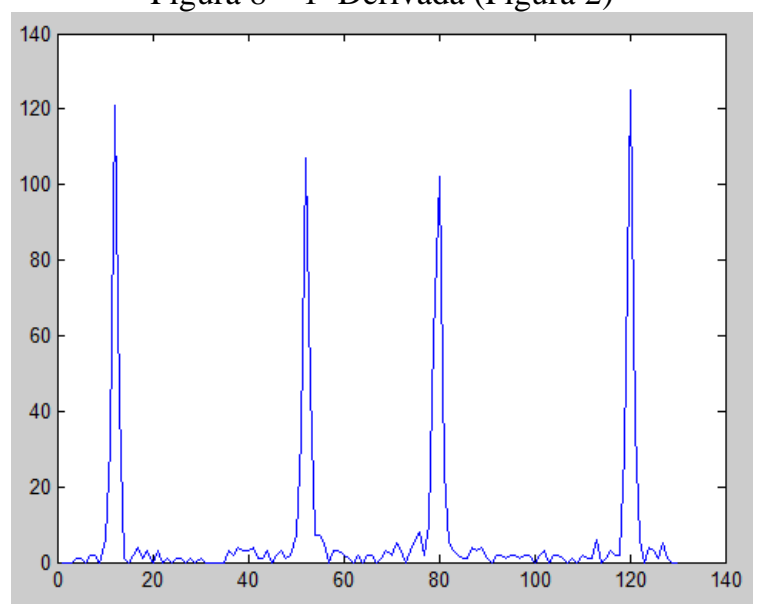

Figura $9-2^{\text {a }}$ Derivada (Figura 2)

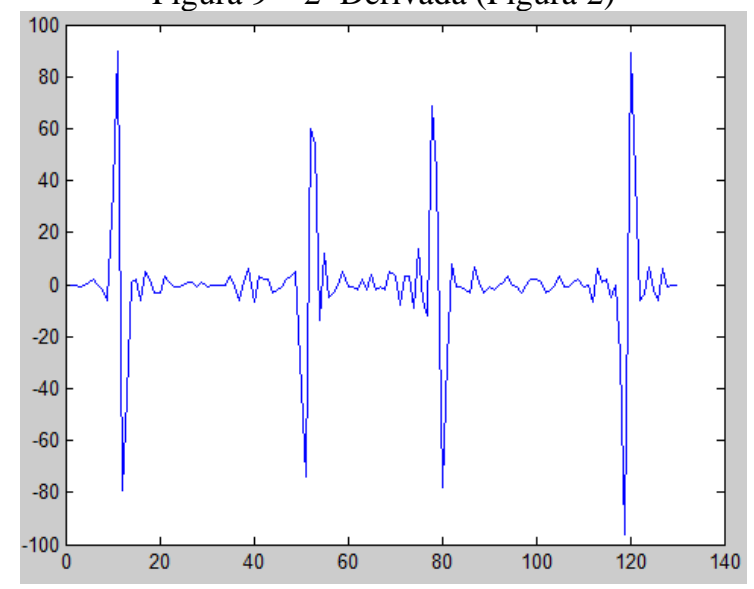

Nas figuras (8) e (9), observa-se a presença de diversos "picos" menores que não correspondem às bordas, assim foram considerados "picos" no caso da $1^{\mathrm{a}}$ derivada aqueles que possuem o valor de, no mínimo, a metade do valor máximo e no caso da $2^{\mathrm{a}}$ derivada aqueles que possuem o valor de, no mínimo, um quarto do valor máximo. 
Por fim, como bordas são elementos de alta frequência, para detectá-las ou realçálas deve-se utilizar filtros do tipo Passa-Alta. $\mathrm{O}$ realce das bordas, em geral, é feito utilizando filtros espaciais lineares que se baseiam no gradiente ou no laplaciano da imagem.

\section{Gradiente}

$$
\nabla f(x, y)=\operatorname{mag}(\nabla \bar{f})=\left[\left(\frac{\partial f}{\partial x}\right)^{2}+\left(\frac{\partial f}{\partial y}\right)^{2}\right]^{1 / 2}
$$

\section{Laplaciano}

$$
\Delta f(x, y)=\left(\frac{\partial^{2} f}{\partial x^{2}}\right)+\left(\frac{\partial^{2} f}{\partial y^{2}}\right)
$$

Os filtros avaliados foram Sobel, Prewitt, Roberts e LoG (Matlab R2010a).

Após a passagem dos filtros, para eliminar possíveis falhas, foi utilizada à operação de fechamento da morfologia matemática. A morfologia matemática "consiste no estudo da forma, isto é, o estudo da estrutura geométrica de entidades presentes em uma imagem [...], seu objetivo é extrair informações relativas à geometria e à topologia de um conjunto desconhecido de uma imagem" [4]. A morfologia matemática fornece um conjunto de operações que permitem que a imagem seja filtrada, corrigida ou retocada sem modificar as características de forma e tamanho dos elementos que restarem após essas operações. A operação de fechamento fecha pequenos espaços que surgem na imagem após a passagem dos filtros [4].

Para realizar a medição, antes é necessário definir o objeto que será medido, para esta operação optou-se que a própria ferramenta computacional iria identificar os elementos presentes na imagem oferecendo ao usuário a opção de escolha de qual elemento a ser medido. As figuras de (10) a (13) apresentam um exemplo desta separação.

Figura 10 - Imagem original

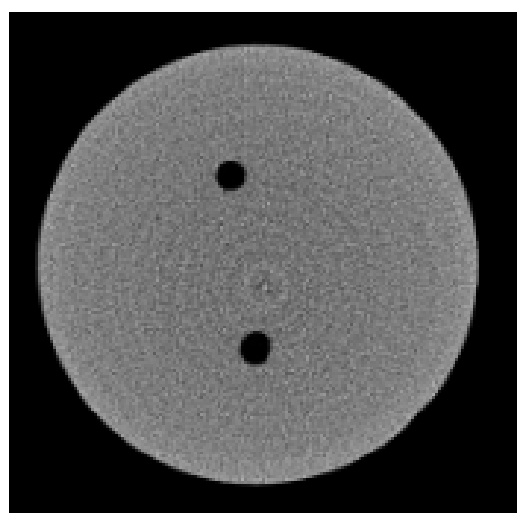

Figura 11 - Elemento 1, círculo externo

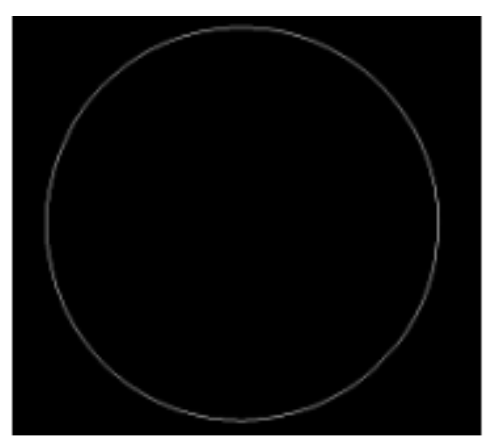


Figura 12 - Elemento 2, fura superior

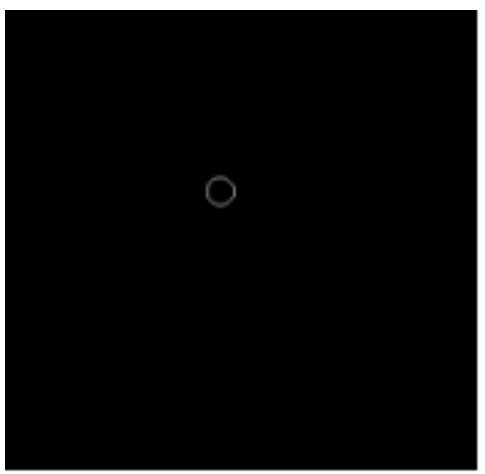

Figura 13 - Elemento 3, fura inferior

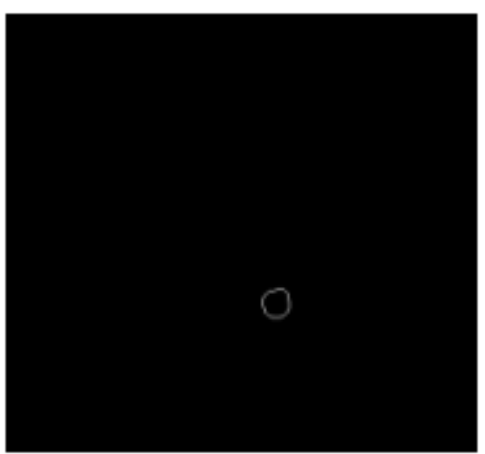

\section{RESULTADOS E DISCUSSÃO}

Para as medições realizadas com a ferramenta computacional foram utilizados blocos cilíndricos padrões. Os resultados foram validados através de comparação com os dados obtidos a partir de medições realizadas pelo SENAI/Nova Friburgo.

$\mathrm{O}$ processo de medição realizado pelo SENAI-NF foi executado manualmente utilizando-se um paquímetro digital. Tomando um bloco cilíndrico (figura 10), foram realizadas medidas do seu diâmetro externo em 3 alturas: superior, centro e inferior. Em cada uma das alturas foram realizadas 4 medidas, nos ângulos de $0^{\circ}, 45^{\circ}$, $90^{\circ}$ e $135^{\circ}$. Os resultados (tabela 1), não necessitaram de arredondamento (com exceção para o cálculo da média), pois os valores foram tomados conforme fornecido pelo próprio instrumento de medição.

Tabela 1 - Medidas realizadas com paquímetro digital

\begin{tabular}{c|ccc}
\hline \multirow{2}{*}{ Ângulo } & \multicolumn{3}{|c}{ Diagonal } \\
\cline { 2 - 4 } & Superior & Central & Inferior \\
\hline 0 0 & 16,86 & 16,85 & 16,84 \\
$45 \circ$ & 16,86 & 16,84 & 16,88 \\
90 o & 16,86 & 16,83 & 16,83 \\
135 o & 16,87 & 16,86 & 16,85 \\
Média & 16,86 & 16,85 & 16,85 \\
\hline
\end{tabular}

No processo computacional, as medidas foram obtidas, a partir de uma fatia, em pixels e depois convertidas para milímetros para isso foi utilizado o tamanho do pixel fornecido pelo tomógrafo que é de $24,77 \mu \mathrm{m}$. No processo de conversão foi necessário a aproximação para 2 casas decimais (número de casas decimais fornecidas pelo paquímetro digital). $\mathrm{Na}$ utilização dos filtros a borda apresentou pequenas falhas. Para a correção, foi utilizado a operação de fechamento da morfologia matemática. Diversos testes foram realizados para se definir o elemento estruturante mais adequado. $\mathrm{O}$ elemento estruturante utilizado foi do tipo disco e tamanho 10. Para realçar visualmente as bordas foi realizado um processo de erosão com o elemento estruturante do tipo disco e tamanho 2 e o 
resultado foi subtraído da imagem "fechada".

Esta última operação não tem influência no resultado sendo apenas para melhor visualização do contorno do objeto.

As figuras de (14) a (18) apresentam as imagens obtidas com as diferentes técnicas avaliadas. Os resultados das medições podem ser observados na Tabela (2).

Figura 14 - Threshold Otsu

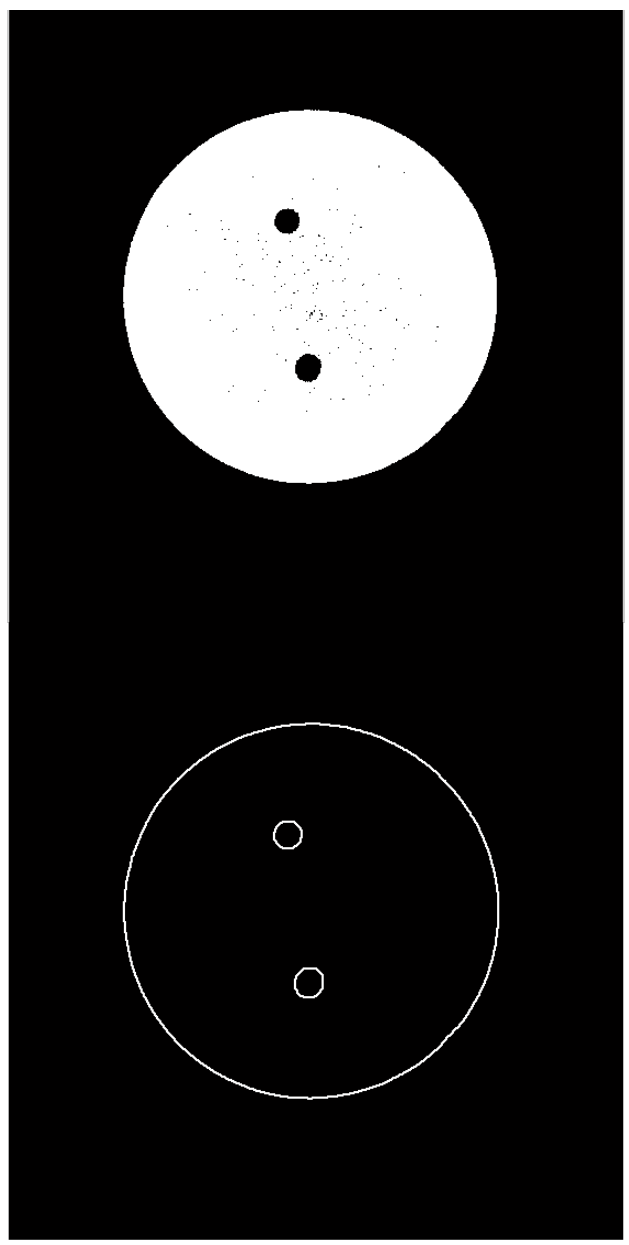

Figura 15 - Filtro de Sobel

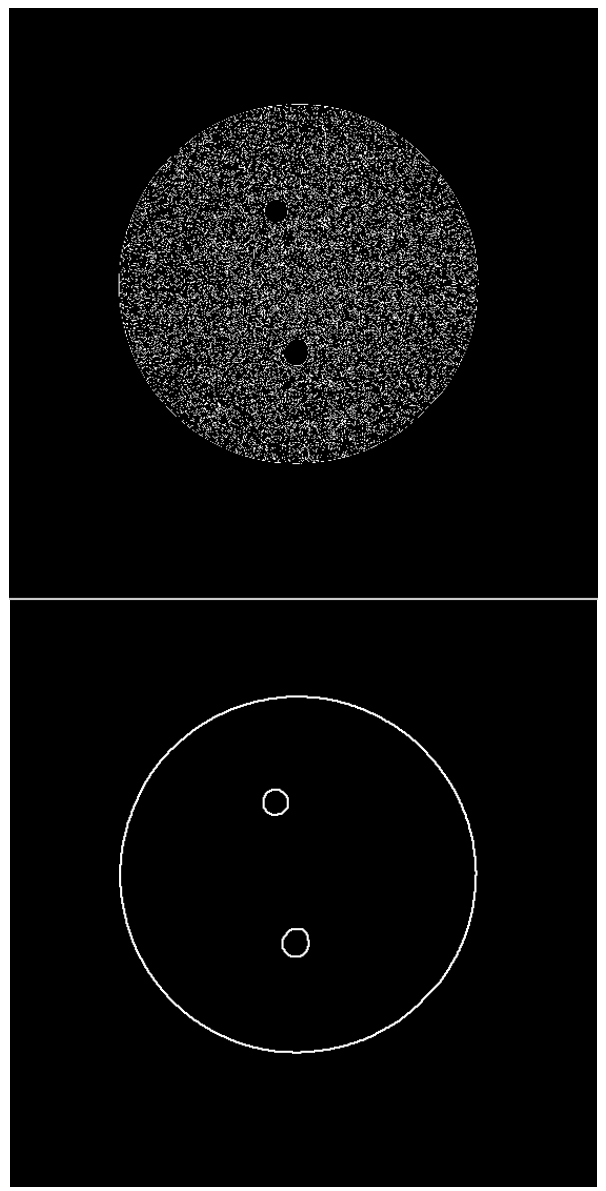

Figura 16 - Filtro de Prewitt

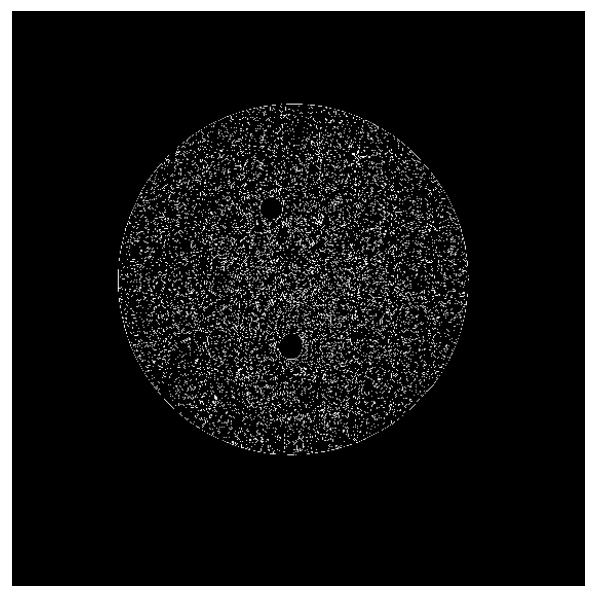




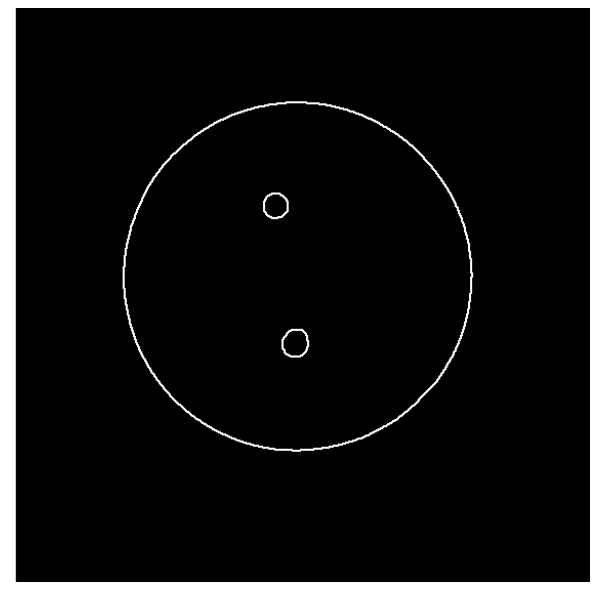

Figura 17 - Filtro de Roberts

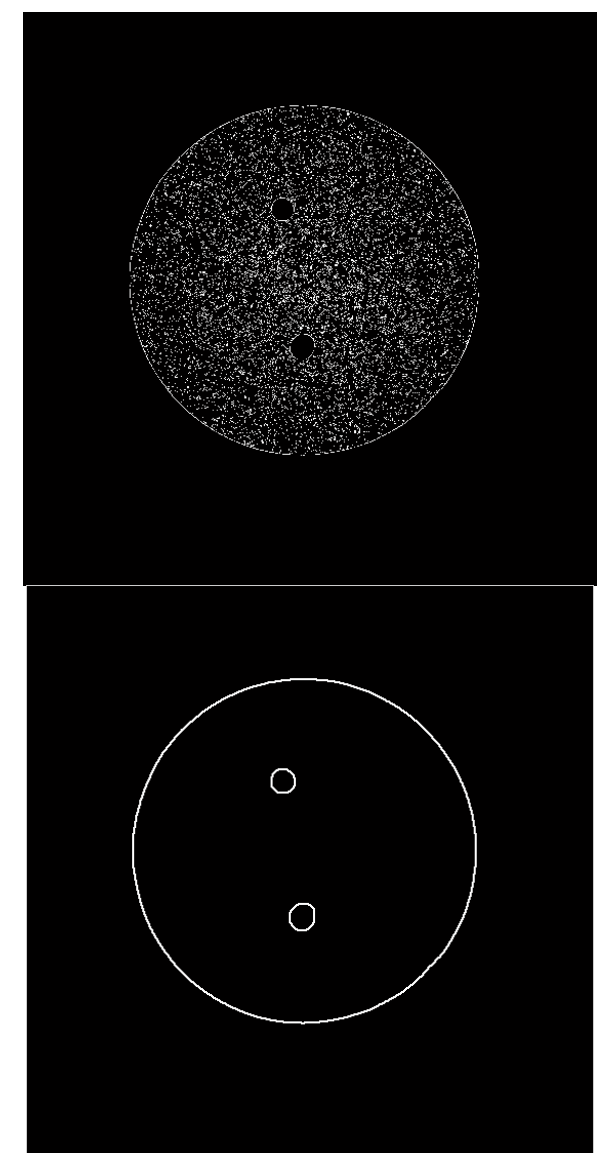

Figura 18 - Filtro LoG Matlab

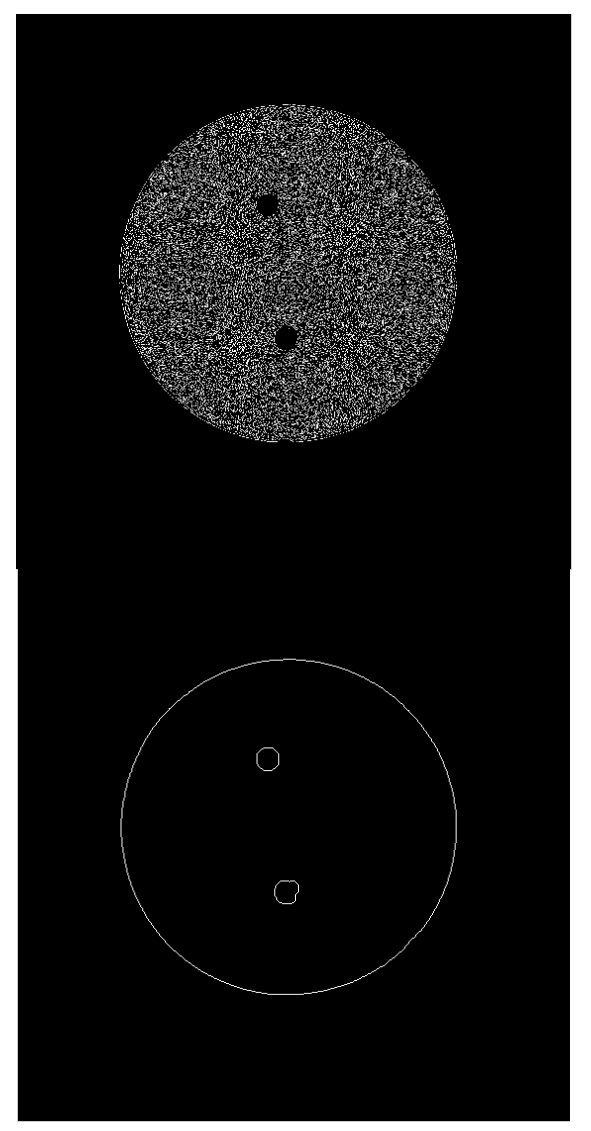

Tabela 3 - Medidas realizadas com a ferramenta computacional

$$
\text { Idos }
$$

no

\begin{tabular}{c|ccccccc}
\hline \multirow{2}{*}{ Ângulo } & \multicolumn{7}{|c}{ Método } \\
\cline { 2 - 8 } & Otsu & Sobel & Prewitt & Roberts & LoG & 1a D & 2a D \\
\hline 09 & 16,79 & 16,82 & 16,82 & 16,82 & 16,79 & 16,82 & 16,84 \\
$45 \varrho$ & 16,89 & 16,89 & 16,89 & 16,92 & 16,89 & 16,92 & 16,94 \\
909 & 16,79 & 16,79 & 16,79 & 16,82 & 16,79 & 16,82 & 16,84 \\
1359 & 16,89 & 16,89 & 16,89 & 16,92 & 16,89 & 16,92 & 16,94 \\
Média & 16,84 & 16,85 & 16,85 & 16,87 & 16,84 & 16,87 & 16,89 \\
\hline
\end{tabular}

Os resultados da ferramenta computacional (tabela 2) apresentaram uma maior dispersão, porém a peça possui um formato ligeiramente oval, apresentando inclusive uma variação ao longo da sua altura. Também não é possível garantir que o as medidas realizadas manualmente (tabela 1), tenham sido realizadas nas mesmas posições, 
(ângulo e altura), em relação ao processo computacional. Pode-se dizer que os resultados obtidos com a $\mu C T$ (tabela 2) são equivalentes aos obtidos com paquímetro digital (tabela 1).

O processo computacional permite a realização de um número de medidas muito superior ao processo manual. As figuras (19) e (20) apresentam, respectivamente, em pixels, o resultado de 1000 medições do diâmetro externo para o mesmo bloco cilíndrico, utilizando a distância entre "picos" da primeira e a segunda derivadas a partir do filtro de Sobel.

\section{Figura 19 - Medidas diâmetro externo ( $1^{\text {a }}$ derivada)}

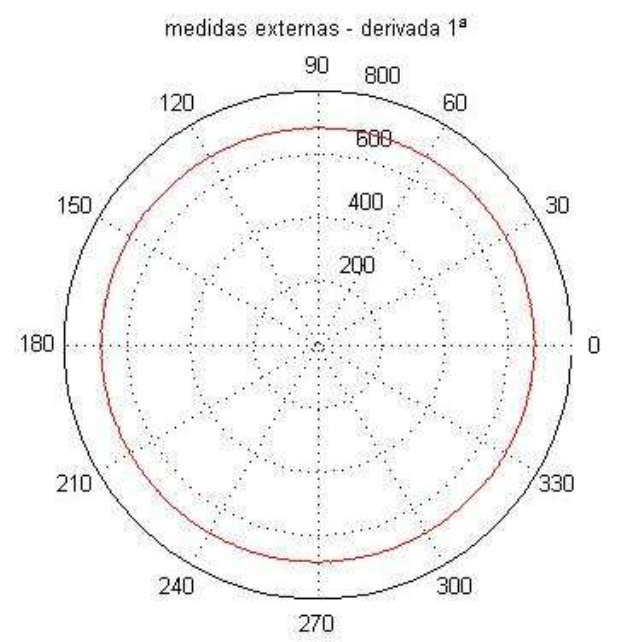

Figura 20 - Medidas diâmetro externo ( $2^{\mathrm{a}}$ derivada)

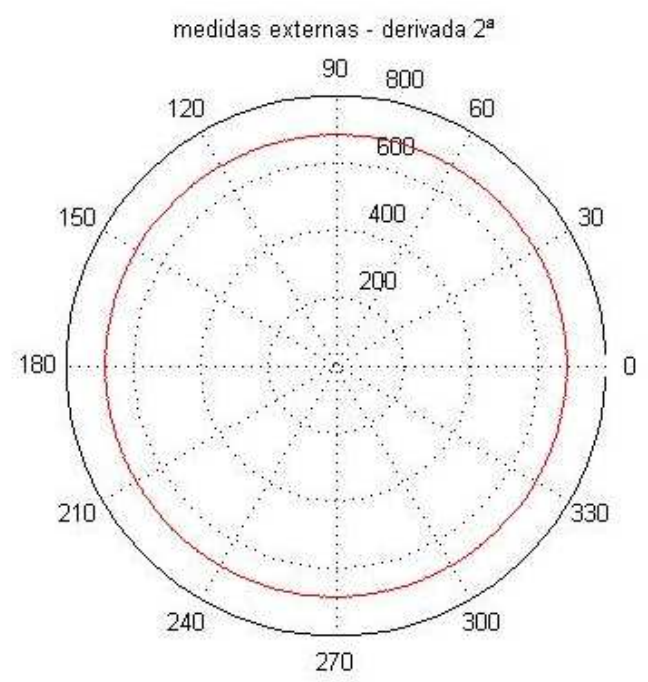

A próxima etapa é a comparação da nuvem de pontos resultante da ferramenta computacional com os dados obtidos com uma máquina de medir por coordenadas.

\section{CONSIDERAÇÕES FINAIS}

O processo manual possui diversas limitações como o tamanho do objeto, a forma, o material e não pode ser utilizado para medidas interiores de forma não destrutiva, além disto, está sujeito a erros sistemáticos. A $\mu \mathrm{CT}$, produz, diversas fatias ao longo do corpo de prova, permitindo, uma análise tridimensional, se necessário.

\section{REFERÊNCIAS}

[1] E. Savio, L. De Chiffre, e R. Schmitt.

Metrology of freeform shaped parts. 
CIRP Annals, vol. 56, no. 2, pp. 810835, 2007.

[2] Kunzler. Maria Regina. Estudo Dos

Desvios Geométricos Gerados na

Obtenção de Biomodelos a Partir de

Imagens Tomográficas. Dissertação

(Mestrado em Metrologia), Universidade

de Santa Catarina, Florianópolis, 2008.

[3] Maques Filho, Ogê e Vieira Neto, Hugo.

Processamento de Imagens. Rio de

Janeiro: Brasport. 1999.

[4] Matta, Wanessa Nascimento.

Metodologia Para Detecção de Máculas

Em Micrografias Utilizando

Morfologia Matemática. Dissertação

(Mestrado em Ciência da Computação) -

UFMG, CNPq, 1998. 93 f.

[5] Nardelli, Vitor Camargo, Medição

Geométrica Utilizando Tomografia

Computadorizada Por Raio-X:

Melhoria do Desempenho Metrológico

Através da Seleção Sistemática dos

Parâmetros de Configuração. Tese

(Doutorado em Engenharia Mecânica),

Universidade de Santa Catarina,

Florianópolis, 2012.

[6] Otsu, N. A threshold selection method from gray-level histograms. IEEE transactions on systems, man and

cybernetics, Tokyo, v.9, n.1, p 62-66, jan. 1979. 